\title{
Management Economics in the Development of Bilibili
}

\author{
Minjie $\mathrm{Liu}^{1, *}$ \\ ${ }^{1}$ Beijing No.80 international high school, Beijing, China, 100027 \\ *Corresponding author. Email: 1520039753@qq.com
}

\begin{abstract}
Management economics is to take the subject of participating in market economy activities as the research goal, and guide the discipline of enterprise management with the help of relevant theories of economics. Management economics shows the prominent characteristics of practical and theoretical close connection, so it is a non-key influencing factor in enterprise management activities. With the rapid development of the social economy, more and more enterprise managers began to slowly realize the importance of management economics. This paper discusses the application of management economics in the management of Bilibili. Management economics helps Bilibili to manage the internal structure of the enterprise in a more detailed way, define his own positioning and make accurate strategic plans. Provide forecast and development assurance for Bilibili development shallow Wells.
\end{abstract}

Keywords: Management Economics, Internet Enterprise, Operating Management

\section{INTRODUCTION}

The rapid development of modern social economy has made the operation and management mode of Internet enterprises innovative and changed to a great extent. In the process of its own development, Bilibili should be good at using modern management theory to ensure a better survival and development in the increasingly fierce market competition environment. Management economics to participate in the market economic activities of the economic and management behavior analysis, the theory of microeconomics and enterprise business development together, guide and help enterprises more scientific and effectively deal with the relationship between their own management and development and the market, at the same time for bilibili innovation reform and production and operation to provide more decision basis, let enterprise management decisions and management activities can truly accord with the actual market development. This paper will study how management economics is applied in the management of Bilibili.

\section{ANALYSIS OF THE REASONS FOR THE SUCCESS OF BILIBILI BASED ON THE PERSPECTIVE OF MANAGEMENT ECONOMICS}

\subsection{Grasp the market situation}

In order to avoid blind operation and management work, enterprises must be fully familiar with the needs of business operation and development, and effectively avoid and control the possible risks, so as to obtain better survival and development in the increasingly fierce market competition environment. The current market economy is changing rapidly, and we must maintain a stable and effective operation and development to continue to move forward.[1]

Bilibili has been focused on original ACG videos. As of June 2019, there were more than 1.8 million UP owners in the B game area, releasing 21 million games, and receiving 60.1 billion views. By reviewing videos and live videos, Bilibili UP owners have changed China's game industry. More than 40 million Chinese fans have included Hanfu, and most of its creators are born in the post-1995s and post-1900s. Young people have become the most enthusiastic group about the inheritance and revival of traditional culture. In 2019, a total of 104 domestic animation works were launched in the national creative area of Bilibili, matching the supply of Japanese animation dramas for the first time;[2] the number of 
monthly active users of Bilibili also exceeded the national drama area for the first time, with the total broadcast time exceeding 300 million hours. Bilibili is one of the largest purchasers of animation rights in China. In 2019, Bilibili invested in and produced 45 Japanese drama animations, accounting for $15 \%$ of the total number of animations produced in Japan. In 2017 and the first half of 2018,312 new Japanese animations were released, of which 201 were broadcast on $\mathrm{B}$, accounting for $64.4 \%$ [2]. In the process of applied management economics, Bilibili actively carries out self-reform and transformation, is good at applying modern management methods, keeping up with the development pace of The Times, and taking scientific and effective means to promote the vitality of enterprises. For example, "for the new generation of young people, the deep cultivation of the seconddimensional entertainment culture". As smartphones become available, the main entertainment tool for young adults at home is mobile phones. Playing games and watching anime videos on mobile phones can also make social friends. Many young people'online social networking became a part of life during the epidemic. The Bilibili is suspended above the video for the live review, namely the bullet screen ". The barrage builds a wonderful syntense relationship, forming a virtual tribal viewing atmosphere, making the Bilibili a cultural community with great interactive sharing and secondary creation. The barrage really changed the Bilibili from a one-way video playback platform to a two-way emotional connection platform. The Bilibili has become a platform for emotional communication among young people who like animation and games. Bilibili is an important secondary yuan game distribution channel in China. It represents more than 500 combined transport mobile games, 13 exclusive agent games, and 1 selfdevelopmentmobile game BBB 1 .

\subsection{Smart allocation of website resources}

The main content of management economics is how to develop and utilize its existing internal resources as far as possible. In the process of participating in market competition activities, find their correct positioning, the beneficiary and beneficiaries from a single group, so that the connection between the two is formed, and finally achieve a win-win situation. In other words, it is softening and integrating sales methods to different levels, and transforming passive consumption into active consumption, so that enterprises can gain more tangible benefits. Bilibili is mainly responsible for background programming and web design. There is content editor responsible for home page topic and online activity planning, some influential online activities such as: January 30,2014 afternoon began webcast "bililibili 2014", video playback exceeded 300000, barrage message exceeded 500000 times, even shake the Chinese New Year's Eve CCTV Spring Festival gala habits. Due to the mature backoffice program, Bilibili does not have to bother typesetting the content of the home page. The videos loved by the audience will automatically appear in a prominent position on the home page, while the low heat will be gradually hidden. Bilibili members had previously established connections by focusing on each other. Similar to Weibo, up's main professional and personalized contributions had formed their own channels to attract specific audiences, and users had also formed their own personalized menu.

\subsection{Diversified content}

A few years ago, the Bilibili party perfectly explained the business concept of "content is king" on Bilibili. Throughout the party. First, choose hot topics, from Which Power to the Wandering Earth to the theme song for 2019. Secondly, reflect the characteristics of the platform. The party kicked off with the cosplay performance of World of Warcraft, with B titles such as Chen Leyi and Daqin KOTO, Jiang Dance Maru. The singing of the League of Legends theme song in 2019 ignited the passionate memories of a generation of games and friendship. Finally, tradition and creativity integrate. Patriotic military music "March of the Iron and Steel Torrent" and patriotic animation song "That Rabbit Those Things" in different forms to express the same love for the motherland. In the 200-minute party, chorus, symphony, antique, electronic music and other music forms. The wonderful ensemble of Mr.Fang Jinlong and orchestra is a gathering between China and many countries, ancient and modern, Chinese and Western, truly achieved music without boundaries. Have "catch shrimp" "for tribe", "outlet to report" second yuan "and" guzheng pipa "" steel torrent "," Disco idol " and other traditional culture compatibility, also have new pants, zhou deep, mayday, Deng Zi chess popular singer balance, realized the harmonious coexistence of creative and traditional, fully embodies the $\mathrm{B}$ multiple inclusive content setting concept. The diverse content has attracted a wider audience, helping Bilibili expand its customer base 


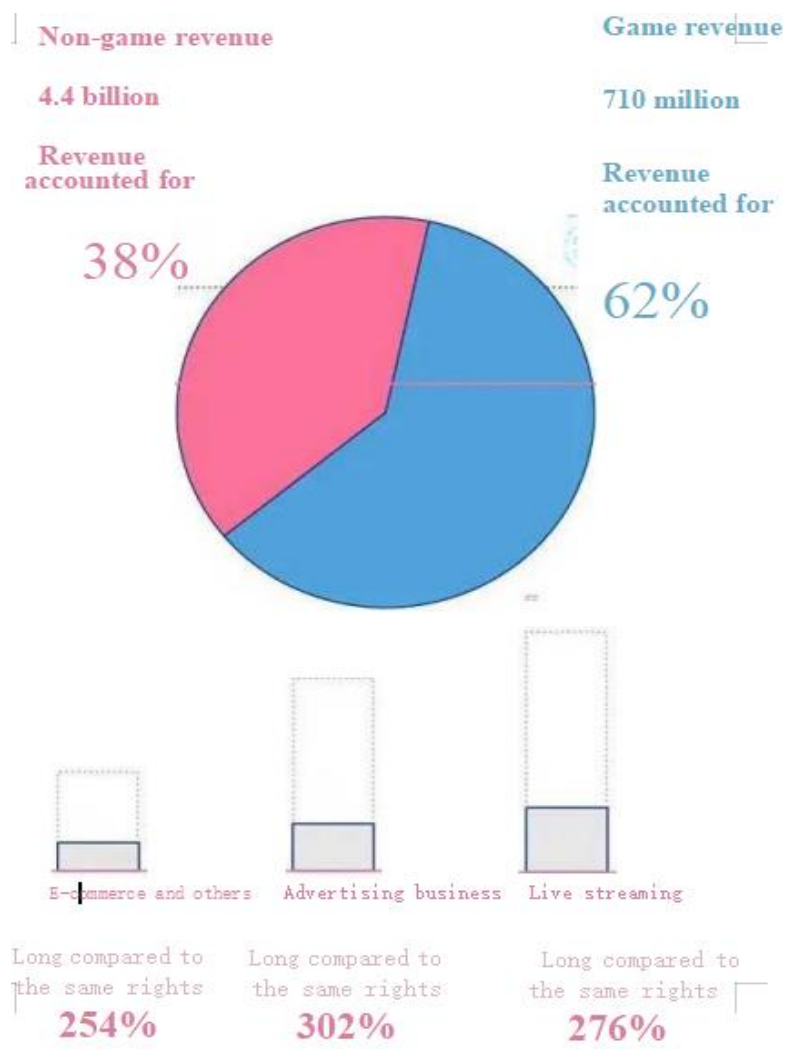

Figure1 Diversified content

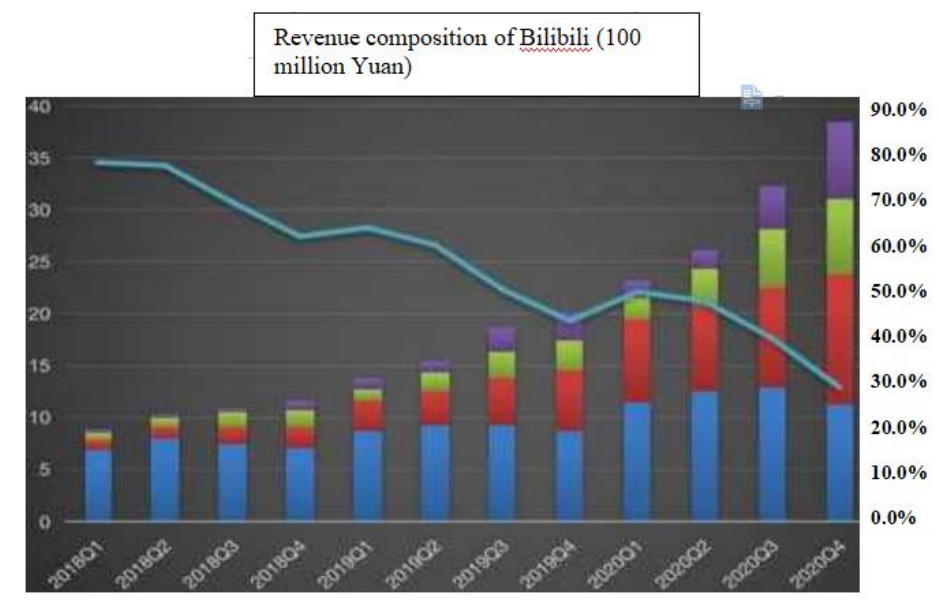

-Mobile games -Value-added services - Advertisement -Electricity - Percentage of games

Figure2 Diversified content

\section{THE DEVELOPMENT PROSPECT OF BILIBILI UNDER THE PERSPECTIVE OF MANAGEMENT ECONOMICS}

\subsection{The "second yuan economy" has come}

The rise and introduction of China's secondary yuan economy from the 1980s to 1990 s has cultivated the first batch of audiences. It has been developed for more than 20 years, and at present, the industry is in a period of rapid development. With the gradual expansion of the secondary yuan industry outstanding talent team, Market segments and business models are also gradually clear, The concept of "quadratic dimension" comes more into view. The perception of the quadratic-dimensional group has also gradually changed. The "quadratic" label is no longer "non-mainstream" and "marginalized". Instead, "with" youth "and" huge consumption potential ". Its main audiences "post-990 s" and "00" are gradually mature and contributing to society.[4]With the growth of the younger generation, the secondary dimensional culture has gradually become an indispensable 
mainstream cultural element in the society. With great development potential. Under the Internet tide, the quadratic element extends from heavy to light users. From core users to mass users, small subculture to the mainstream tide culture. This is also the path of many pop culture phenomena in the past (such as online shopping, which sold less than 1,000 this year 10 years ago). In this era, mobile Internet and pan-entertainment trends will play a key role in driving them. There is another trend, the drivers of social interaction will become increasingly important. Tencent has two of China's largest social networking platforms, WeChat and QQ. Secondary dimension has a strong circle culture and social attributes, social can in turn promote the generation of quadratic content. If China can really rise with the revival of China's economy, the most profitable station must be B. Set a small goal, if Bilibli develops into the largest or even the largest animation production base in the country, then the cultural value and economic value created by Bilibili cannot be measured.

\subsection{Uncertainty brought about by the business expansion}

Bilibili started as the second yuan, but now the content is more and more, and the core value of bullet screen-barrage is that the information is two-way transmission. The feeling of watching with the barrage is a bit like watching with a lot of people, and this feeling of "integrating into the group" is actually a basic psychological need of people. To tell the truth, for the three typical East Asian countries, China, Japan and South Korea, the life pressure is getting greater and greater, and the existence of the barrage itself is in line with the trend of The Times. In terms of quantity, the mobile games represented by Bilibili are obviously not comparable with the number of game giants such as Tencent netease; in terms of traffic is certainly not comparable with the national mobile games such as pesticide eating chicken. However, Bilibili they have a very clear positioning, mainly focusing on the ACG agent and research and development of mobile games. At present, Bilibili has four commercial modes, namely mobile game, advertising, live broadcast and value-added services and others. Among them, mobile game accounted for $83.4 \%$ of B's net revenue in 2017, while the two popular mobile games, Fate/Grand Order and Blue Line, have contributed $71.8 \%$ and $12.7 \%$ of BBB 1 revenue, respectively. Arguably, Bilibili has achieved such a good result in 2017, almost directly linked to Fate/Grand Order's success.[5] But commercially, a single revenue structure is almost synonymous with high risk; moreover, B is a single revenue structure under a single game. As Fate/Grand Order life cycle gradually moves to middle and late, it is suspense whether B can find the same strong game for Fate/Grand Order, itself.

\section{CONCLUSION}

Now, Bilibili has received strategic investment from Tencent, Alibaba and SONY. The latest market value of Bilibili is 8.803 billion yuan, which is one step closer to the target of 10 billion dollars.[6] A detailed study of Bilibili shows that the success of Bilibili is firstly the success of internal control of development strategy. There are two main reasons: 1 . Clear strategic content: focus on ACG and share original videos 2. Strategic customer precision: for the new generation of young people, deeply cultivate the entertainment culture of the second yuan. Moreover, even under the attack of PGC and short video, Bilibili still relies on vertical to create a strong field, deeply cultivate the vertical field, further strengthen the advantageous works, and then negotiate with game vendors with content as a bargaining chip. Second, Bilibili's refined operations. Bilibili was not strong in content except quadratic elements, but it successfully rose under the operation of Bilibili and formed the most concentrated content base in the whole network.

\section{REFERENCES}

[1] Wang Jiaxia. China Collective Economy,2021(19):59-60. (in Chinese)

[2] internal control case analysis | B standing success is the development strategy of success.(September4).https://baijiahao.baidu.com/? $\mathrm{id}=1663592837953866074 \& \mathrm{wfr}=$ spider $\&$ for $=\mathrm{pc}$

[3] Li Yuanyuan. Analysis on "Breaking circle" Strategy of STATION B [J]. New Media Research (14):2.

[4] Wang Cheng, $\mathrm{Xu}$ Lin. Modern Enterprise,2018(08):94-95. (in Chinese)

[5] ChenHan peony. "introduction to development prospects - B B stop iron powder and ordinary students in perspective." bi li bi li $\left(^{\circ}{ }^{\circ} \supset \square\right.$ cheers - bilibili, www.bilibili.com/read/cv616326/. Accessed 2 Sept., 2021.

[6] What is the future of Station B after the successful lap breaking?. (September 5) _ panoramic panoramic mesh. https://weyt.p5w.net/article/2404423 